\title{
A Reverse Phase HPLC Method Development and Validation for the Determination of Paliperidone in Pure and Dosage Forms
}

\author{
K.UMAMAHESSWAR, G.RAMU and C.RAMBABU* \\ Department of Chemistry, Acharya Nagarjuna University, Nagarjuna Nagar, Guntur, India \\ rbchintala@gmail.com
}

Received 20 June 2012 / Accepted 8 July 2012

\begin{abstract}
A simple reverse phase HPLC method was developed and validated for the assay of paliperidone in pure and tablets. The analysis was carried out on Shimadzu HPLC model equipped with inertsil ODS C-18 column, LC-10AT pump, variable wavelength programmable UV-Detector SPD10AVP and rheodyne injector with $20 \mu \mathrm{L}$ fixed loop. Acetonitrile and methanol in the ratio 10:90 v/v at a flow rate of $1.0 \mathrm{~mL} / \mathrm{min}$ was employed for elution and the components were detected at $235 \mathrm{~nm}$ at ambient temperature. The method was validated as per the ICH guidelines. The retention time for paliperidone was found to be $5.67 \mathrm{~min}$. Linearity was observed in the concentration range of 20-120 ppm with correlation coefficient 0.9990 . The percent of relative standard deviation of six replicate measurements was found to be 1.25 indicate that the proposed method was precise. Recovery studies were conducted at three different concentration levels within the linearity limits and the average percent of recovery was 99.56. The amount of paliperidone drug content present in tablet dosage form was determined and found to be $99.80 \%$. Therefore, the developed method was simple, precise and accurate and can be successfully applied for the estimation of paliperidone in pharmaceutical dosage forms
\end{abstract}

Keywords: Paliperidone, UV detection, Method Validation, Linearity and Assay

\section{Introduction}

Paliperidone (Figure 1) belongs to the class of atypical antipsychotics and is the active metabolite of a well-known active substance risperidone. It is one of the most commonly used atypical antipsychotic medication ${ }^{1-3}$. Risperidone is extensively metabolized to 9hydroxy- risperidone (i.e. paliperidone) via CYP2D6 and the exposure after administration of risperidone is often presented in terms of "active moiety", which is the sum of risperidone and 9- hydroxy- risperidone plasma levels. Paliperidone (9-OH-risperidone) is a receptor monoaminergic antagonist that exhibits the characteristic dopamine type 2 (D2) and serotonin (5-hydroxytryptamine 5-HT) type 2A (5-HT2A) antagonism of antipsychotic drugs $^{4-6}$. The chemical structure of the drug is presented in Figure 1. An extensive literature survey is carried out and found some LC-MS/MS methods ${ }^{7-8}$, HPTLC methods ${ }^{9-10}$, HPLC method $^{11}$ and a UPLC method ${ }^{12}$ for the determination of risperidone and the enantiomers of 9-hydroxyrisperidone in plasma, urine pharmaceutical formulations respectively. 


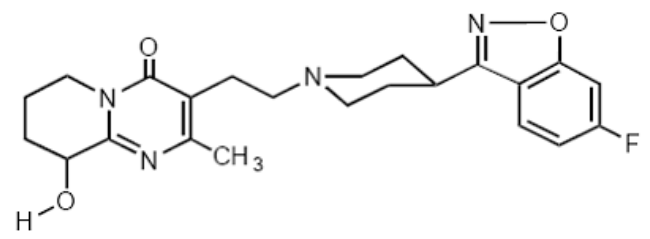

\section{Experimental}

Figure 1. Structure of paliperidone

\section{Instrumentation}

The analysis of the drug was carried out on Shimadzu HPLC model (VP series) containing LC10AT (VP series) pump, variable wavelength programmable UV/Visible detector SPD-10AVP and rheodyne injector (7725i) with $20 \mu \mathrm{L}$ fixed loop. Chromatographic analysis was performed using Inertsil ODS C-18 column with 250 x $4.6 \mathrm{~mm}$ internal diameter and $5 \mu \mathrm{m}$ particle size.

\section{Materials and Methods}

Paliperidone in pure form was a gift sample obtained from R.V Labs, Guntur. Tablets of paliperidone were procured from the local market. Solvents such as acetonitrile, methanol and water of HPLC grade were purchased from E.Merck, Mumbai, India. The mobile phase was prepared by mixing acetonitrile with methanol in the ration 10:90 (v/v) and was degassed by sonicating for 5 min before use. The stock solution of paliperidone $(1 \mathrm{mg} / \mathrm{mL})$ was prepared by transferring $10 \mathrm{mg}$ of the pure drug in $10 \mathrm{~mL}$ of the diluents and sonicated for five minutes to dissolve and filtered through $0.2 \mu \mathrm{m}$ Ultipor filter paper. Further $0.8 \mathrm{~mL}$ of the stock was diluted to $10.0 \mathrm{~mL}$ to get a working standard $80.0 \mu \mathrm{g} / \mathrm{mL}$. Four tablets of paliperidone (INVEGA-9 mg) were crushed to give finely powdered material. An amount of powder equivalent to $10 \mathrm{mg}$ of the drug was accurately weighed and dissolved in $5 \mathrm{~mL}$ of methanol in a $10 \mathrm{~mL}$ of volumetric flask sonicated to dissolve the drug and then filtered through Ultipor $\mathrm{N}_{66}$ Nylon 6, 6 membrane sample filter paper. Finally the volume of the filtrate was adjusted to the mark with the same solvent to obtain concentration of $1 \mathrm{mg} / \mathrm{mL}$. $80.0 \mu \mathrm{g} / \mathrm{mL}$ working sample was prepared by measuring $0.8 \mathrm{~mL}$ of the sample and transferred into a $10 \mathrm{~mL}$ volumetric flask and made up to the mark.

\section{HPLC operating conditions and suitability parameters}

The HPLC system and column were kept at ambient temperature. The column was equilibrated for $30 \mathrm{~min}$ with the mobile phase flowing through the system at a flow rate of $1.0 \mathrm{~mL} / \mathrm{min}$. The detection wavelength was set at $235 \mathrm{~nm}$. A volume of $10 \mu \mathrm{L}$ of the standard was injected into the system and the components eluted from the system were monitored for a run time $10 \mathrm{~min}$. A sharp peak was found to be at $5.68 \mathrm{~min}$ with 4454 theoretical plate count and 1.91 tailing which were within the limits of acceptance. The system suitability parameters of the proposed method were presented in Table 1. Typical liquid chromatograms for the standard and sample were presented in Figure 2 and 3

Table 1. System suitability parameters of paliperidone

\begin{tabular}{lc}
\hline Parameters & Values \\
\hline Retention time & 5.67 \\
Theoretical plates & 4454 \\
Tailing factor & 1.91 \\
Resolution & Not applicable \\
\%RSD & 1.256 \\
\hline
\end{tabular}




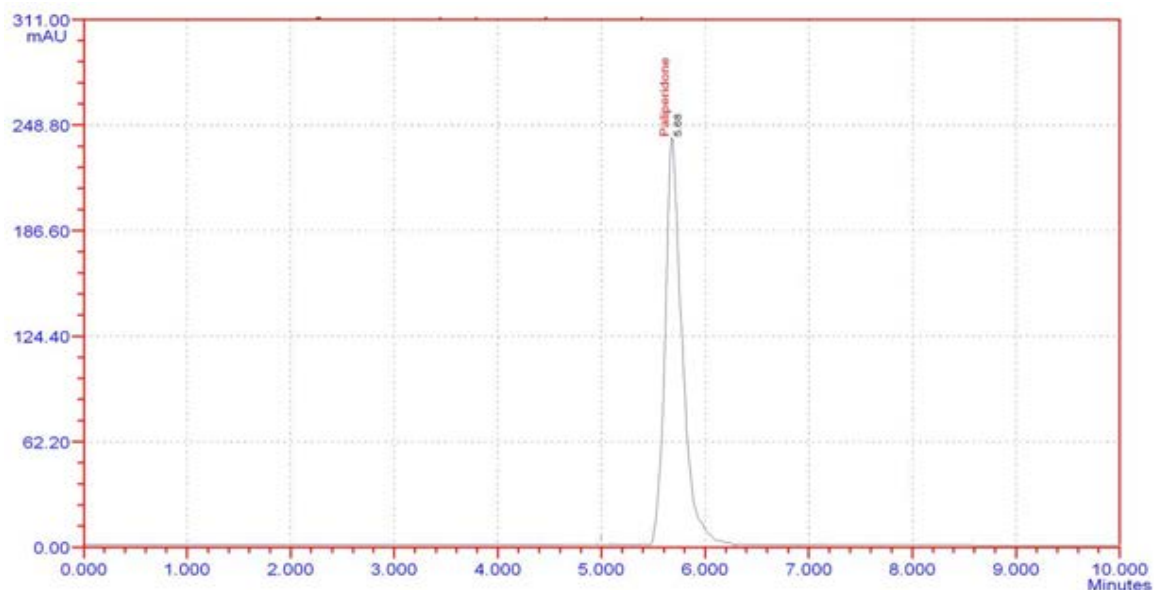

Figure 2. A typical chromatogram of paliperidone standard

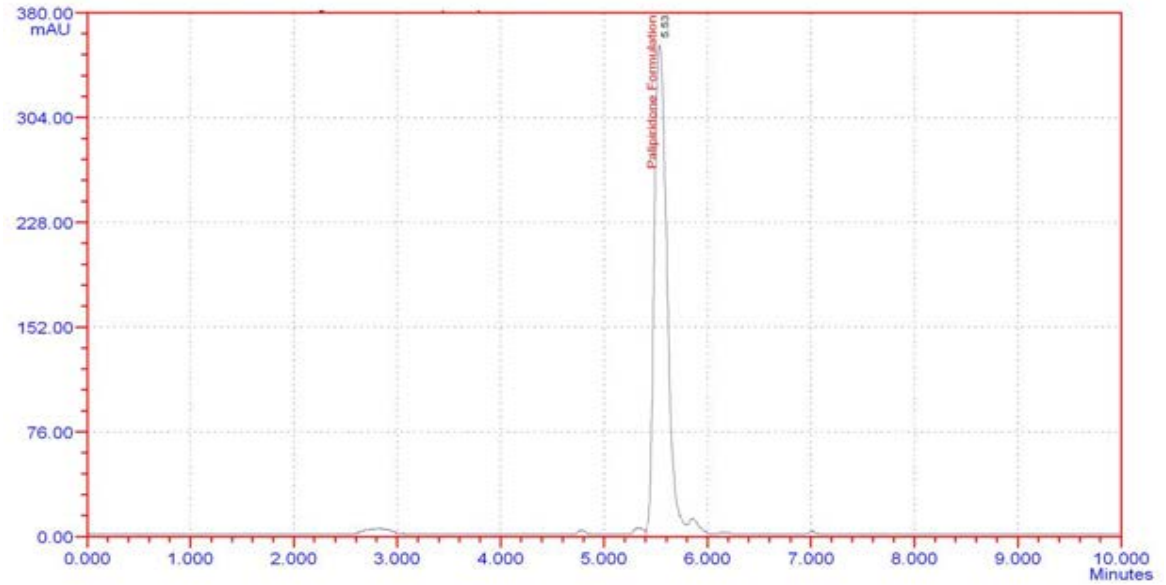

Figure 3. A typical chromatogram of paliperidone formulation

\section{Method Validation}

\section{Precision}

Repeatability of the method was checked by injecting six replicate injections of $80 \mu \mathrm{g} / \mathrm{mL}$ working standard solution of paliperidone on the same day as intraday precision study. Statistical analysis was carried out to find standard deviation and percent of relative standard deviation (\%RSD) of the peak areas and found to be 3611.25 and 1.25 respectively and the values were given in Table 2.

Table 2. Precision parameters of paliperidone

\begin{tabular}{lccrc}
\hline Name of the drug & Concentration & Injection ID & Peak Area & Statistical Report \\
\hline & & 1 & 290720 & \\
Paliperidone & 2 & 287693 & Average $=287560$ \\
& \multirow{2}{*}{$80 \mu \mathrm{g} / \mathrm{mL}$} & 3 & 285027 & S.D. $=3611.25$ \\
& & 4 & 284495 & \%R.S.D. $=1.256$ \\
& 5 & 284428 & \\
\hline
\end{tabular}




\section{Linearity}

A series of different concentrated working standard solutions of paliperidone in the concentration range of 20 to $120 \mu \mathrm{g} / \mathrm{mL}$ were injected into the chromatographic system. The chromatograms were developed and the peak area was determined for each concentration of the drug solution. Calibration curve was obtained by plotting the peak area versus the concentrations of paliperidone and it was given in Figure 4. The plot was found to be linear with slope, intercept and correlation coefficient was found to be 3536.0,3589 and 0.9994 respectively. Limit of detection (LOD) and limit of quantification (LOQ) were calculated as per ICH guidelines and found to be 0.2 and $0.5 \mu \mathrm{g} / \mathrm{mL}$ respectively. The linearity studies were reported in Table 3.

Table 3. Linearity studies of paliperidone

\begin{tabular}{cccl}
\hline S.No. & Concentration, $\mu \mathrm{g} / \mathrm{mL}$ & Area of the peak & Linear regression analysis report \\
\hline 1 & 20.0 & 78313 & Linearity $=20-120 \mu \mathrm{g} / \mathrm{mL}$ \\
2 & 40.0 & 143678 & Slope $=3536$ \\
3 & 60.0 & 214276 & Intercept $=3589$ \\
4 & 80.0 & 293611 & Correlation Coefficient $=0.9990$ \\
5 & 100.0 & 354336 & LOD $=1.25 \mu \mathrm{g} / \mathrm{mL}$ \\
6 & 120.0 & 426081 & LOQ $=4.0 \mu \mathrm{g} / \mathrm{mL}$ \\
\hline
\end{tabular}

\section{Accuracy}

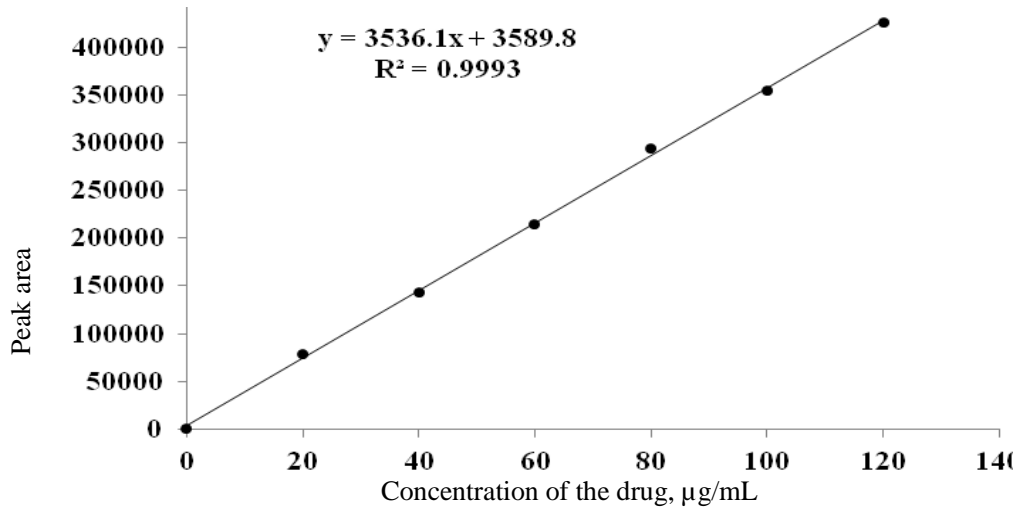

Figure 4. Calibration plot of paliperidone

The accuracy of the method was determined by calculating recovery of paliperidone by the method of standard addition. A known amount of paliperidone was added to a pre quantified sample solution and the amount of paliperidone was estimated by measuring the peak area. The recovery studies were carried out at three different concentrations 40, 80 and 120 $\mu \mathrm{g} / \mathrm{mL}$ each concentration was analyzed three times and amount of paliperidone was estimated by measuring the peak area by fitting these values to the straight line equation of calibration curve. The mean percent of recovery was found to be 99.56. The results were presented in Table 4.

\section{Assay analysis of formulations}

The tablets of the drug were analyzed by the proposed method, the percent of drug present in the tablet was found and the results were presented in Table 5. The percent of recovery was within the limits which show specificity of the method towards the excipients that were present in the tablet. 
Table 4. Accuracy of the proposed method at three different concentrations

\begin{tabular}{cccc}
\hline Spike level & Concentration, $\mu \mathrm{g} / \mathrm{mL}$ & \% of Recovery & Statistical analysis \\
\hline \multirow{3}{*}{$50 \%$} & 40 & 98.35 & Average $=99.49$ \\
& 40 & 99.47 & S.D. $=1.155$ \\
& 40 & 100.66 & \%R.S.D. $=1.161$ \\
$100 \%$ & 80 & 99.28 & Average $=99.19$ \\
& 80 & 98.13 & S.D. $=1.027$ \\
$150 \%$ & 80 & 100.18 & \%R.S.D. $=1.035$ \\
& 120 & 99.66 & Average $=100.0$ \\
& 120 & 100.2 & S.D. $=0.3010$ \\
& 120 & 100.16 & \%R.S.D. $=0.3010$ \\
& & & Over all statistical analysis \\
& & & Average $=99.56$ \\
& & & S.D. $=0.8638$ \\
& & & \%R.S.D. $=0.8670$ \\
\hline
\end{tabular}

Table 5. Assay results of formulation

\begin{tabular}{cccc}
\hline Formulation & Labeled claim, $\mathrm{mg}$ & Amount found, $\mathrm{mg}$ & \% Paliperidone \\
\hline INVEGA Tablet & $9.0 \mathrm{mg}$ & $8.98^{*}$ & 99.80 \\
\hline \multicolumn{4}{c}{ *Average of five determinations }
\end{tabular}

\section{Robustness and Ruggedness}

To determine the robustness of the method, one parameter from the optimized chromatographic conditions was varied keeping other conditions at constant. The effect of small change in flow rate, percent of composition of mobile phase, wavelength were studied. In the study of ruggedness six replicate injections of standard and sample solutions were analyzed and inter day precision was calculated under different columns, different instruments and on three different days over a period of one week. Robustness and ruggedness were also expressed in terms of percentage relative standard deviation. From the study of robustness and ruggedness it was evident that there was no considerable effect on the recovery.

\section{Results and Discussion}

The nature of the sample, its molecular weight and solubility decides the proper selection of the stationary phase. The drug paliperidone being a polar compound and its structure is given in Figure 1 preferably analyzed by reverse phase technique. The elution of the compound from the column was influenced by polar mobile phase. The concentration of the methanol and acetonitrile were optimized to give symmetric peak with short run time based on asymmetric factor and peak area obtained. Different mobile phases were tried but satisfactory separation, well resolved and good symmetrical peaks were obtained with the mobile phase acetonitrile: methanol in the ratio of 10:90 (v/v). The retention time of paliperidone was found to be $5.68 \mathrm{~min}$, which indicates a good base line. Atypical chromatogram of the standard and sample solution of paliperidone were presented in Figure 2 and 3.The \%RSD values for accuracy and precision studies obtained were found to be less than $2 \%$ which revealed that developed method was accurate and precise. The results were presented in Table 1 and 2. The developed method was linear within the range of concentration 20.0-120.0 $\mu \mathrm{g} / \mathrm{mL}$ with good correlation between area of the peak and concentration of the drug. The calibration plot was given in Figure 4. The results of linear regression analysis were presented in Table 3 . The accuracy of the proposed method was 
ascertained by determining the percent of recovery at three different concentration levels and the results were presented in Table 4. The Proposed method was applied for the determination of paliperidone in tablet formulation and result for paliperidone was comparable with the corresponding labeled amount presented in Table 5. The absence of additional peaks indicates no interference of the excipients.

\section{Conclusion}

The proposed method is simple, rapid, accurate, precise and specific. Its chromatographic run time of 10 min allows the analysis of a large number of samples in short period of time. Therefore, it is suitable for the routine analysis of paliperidone in pharmaceutical dosage form.

\section{Acknowledgement}

The authors are very much thankful to R.V Laboratories, Guntur for providing laboratory facilities.

\section{References}

1. Sandra B, Krishna T, Luc J, Bart R, Marc D M, Stefaan R, Nancy van O, Marielle E, and Adriaan C, J Clin Pharmacol., 2009, 49 (11), 1318.

2. Owen RT, Drugs Today, 2007, 43(4), 249.

3. Green and Ben, Paliperidone: A clinical review, Current Drug Therapy. 2009, $4,7$.

4. Kane J, Canas F, Kramer M, Ford L, Gassmann-Mayer C, Lim P and Eerdekens M. Schizophr Res., 2007, 90(1-3), 147.

5. Christian D, Michael N and Zachariah D, American Journal of Health System Pharmacy, 2008, 65 (5), 403.

6. Canuso C M, Youssef E A, Bossie C A, Turkoz I, Schreiner A and Simpson G M. Int Clin Psychopharmacol., 2008, 23 (4), 209.

7. Marc De Meulder, Bart M M, Remmerie, Ronald de Vries, Luc L A, Sips, Sandra Boom, Edwin W J. Hooijschuur, Nico C, van de Merbel and Philip M M B L, J Chromatogr B., 2008, 870 (1), 8.

8. $\quad$ Manickam A and Stephen R M, J Mass Spectrom., 2000, 35(6), 718.

9. Shubhangi M, Pawar1 and Sunil R Dhaneshwar, J Pharm Biomed Sci., 2012, 16 (15), 54.

10. Rashmin B Patel, Mrunali R. Patel, Kashyap K. Bhatt and Bharat G Patel, Anal Methods, 2010, 2, 525.

11. Sanjay A Jadhav, Chromatogr Res Int., 2011, 10, 1.

12. Hima Bindu K, Nitin Haridas Dhekale, Suryanarayana M V and Anjaneyulu Y, $J$ Liquid Chromatogry \& Related Technol., 2012, 35(4), 5. 